\title{
Chương trình máy tính Việt bayesvl chính thức xuất bản trên CRAN
}

Phòng Khoa học \& Công nghệ, Trường Đại học Phenikaa

Hà Nội, 30-05-2019

Ngày 24-05-2019, chương trình máy tính “bayesvl” chạy trên môi trường $\mathrm{R}$ (ngôn ngữ lập trình và môi trường phần mềm miễn phí phục vụ cho tính toán thống kê và đồ họa) do Trung tâm Nghiên cứu Khoa học Xã hội liên ngành (ISR), Trường Đại học PHENIKAA thiết kế và phát triển chính thức được xuất bản trên CRAN - thư viện lưu trữ, phân phối các phiên bản chính thức của $\mathrm{R}$ [1]. Phần mềm được xuất bản đánh dấu cột mốc quan trọng cho hành trình hơn 18 tháng lên ý tưởng, thiết kế và triển khai của Trung tâm ISR. (Mã nguồn mở trên Github và CRAN [1, 2])

Từ cuối năm 2017, Trung tâm ISR đã bắt đầu nghiên cứu khả năng ứng dụng thống kê Bayesian và các cải tiến của nó so với thống kê truyền thống cho Khoa học xã hội và nhân văn (KHXHNV). Bayesian là một cách tiếp cận mới đối với các nhà KHXHNV trên thế giới, vì thế sử dụng phần mềm này rộng rãi vẫn còn gặp nhiều khó khăn do sự phức tạp của các yếu tố toán học và code máy tính. Với mong muốn góp phần cải tiến phương pháp khoa học [3], đồng thời hướng tới xu hướng Open Science (khoa học mở) [4], sử dụng hiệu quả chi phí nghiên cứu [5], Trung tâm ISR đã xây dựng chương máy tính 'bayesvl' trên môi trường R. Phần mềm bayesvl được xây dựng hướng đến mục tiêu sư phạm, hỗ trợ người tiếp cận và sử dụng thống kê Bayesian thông qua xây dựng, thiết kế mô hình lưới Bayesian từ dữ liệu, tiến hành mô phỏng $\mathrm{MCMC}$, thực hiện kiểm tra thông số kĩ thuật và kết quả bằng hình ảnh.

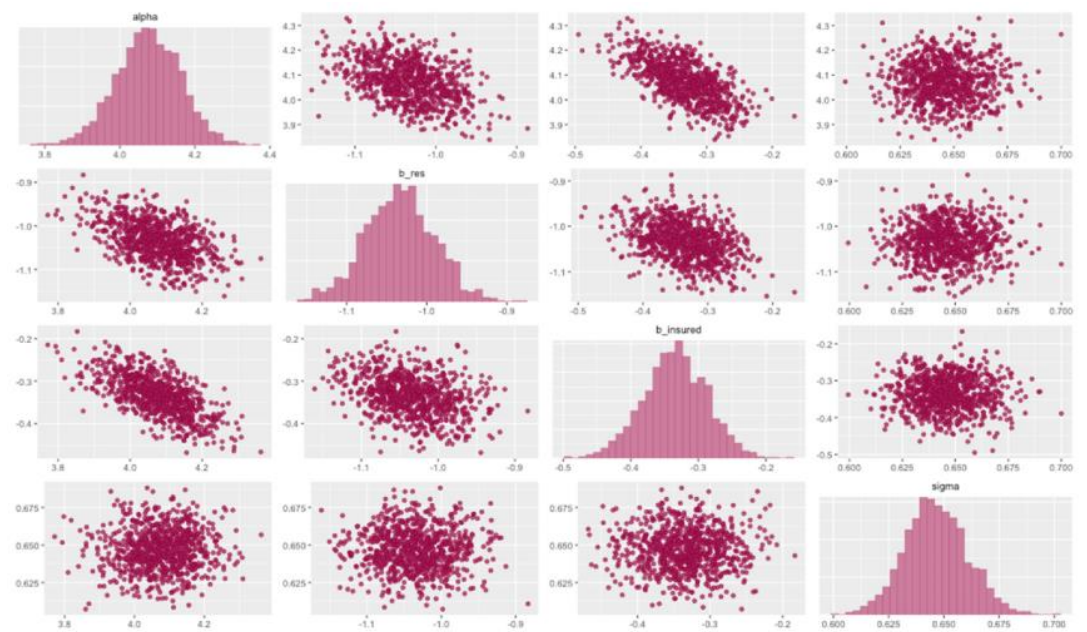

Hình ảnh được sản xuất bởi chương trình bayesvl [6] 
Theo TS. Vương Quân Hoàng - Giám đốc Trung tâm ISR: “Trong nghiên cứu khoa học, việc xuất bản chương trình máy tính cũng mang ý nghĩa công bố phương pháp khoa học và thuật toán ứng dụng cho các nhà khoa học khác cùng sử dụng. Ý nghĩa của nó không giới hạn ở việc công bố thông tin khoa học như bài báo khoa học bình thường. Với tính ứng dụng cao, bayesvl có tiềm năng trợ giúp nhiều nghiên cứu khoa học trong tương lai. Chính thức được chấp thuận xuất bản trên CRAN, bayesvl là phần mềm mở và miễn phí hoàn toàn trên môi trường $\mathrm{R}$ rất thông dụng giúp các nhà KHXHNV sử dụng một cách dễ dàng, gia cố sức mạnh cho phương pháp luận của lĩnh vực KHXHNV trong thời đại công nghệ 4.0".

Áp dụng vào thực tế, năm 2018, nhóm nghiên cứu của trung tâm ISR đã sử dụng thống kê Bayesian trong nghiên cứu Cultural Additivity [7], được xuất bản trên tạp chí Palgrave Communications thuộc Nature Research (tháng 12/2018). Năm 2019, các phần của thuật toán 'bayesvl' tiếp tục được sử dụng trong Preprint về tiếp biến văn hóa (đang peer review) [8] và 2 công bố của Trung tâm ISR trên tạp chí Data [ESCI; Scopus] [6, 8].

Đối với KHXH Việt Nam và có thể là cả châu Á, hiện nay việc đóng góp phương pháp luận và thuật toán vẫn còn là điều hiếm. Chính vì vậy, bayesvl với tính sư phạm trong áp dụng phương pháp thống kê Bayesian là đóng góp quan trọng của Trường Đại học PHENIKAA cho khoa học thế giới, nhất là khi thống kê Bayesian đang được giới khoa học quốc tế quan tâm.

Note: Published in Phenikaa University website: http://phenikaauni.edu.vn/chitiet/su-kien/phan-mem-viet-bayesvl-chinh-thuc-xuat-ban-tren-cran

\section{References:}

[1] La, V. P., \& Vuong, Q. H. (2019). bayesvl: Visually Learning the Graphical Structure of Bayesian Networks and Performing MCMC with 'Stan'. The Comprehensive $R$ Archive Network (CRAN): <https://cran.rproject.org/web/packages/bayesvl/index.html>; version 0.8.5 (May 24, 2019).

[2] Vuong, Q. H., La, V. P. (2019). BayesVL package for Bayesian statistical analyses in R. Github: <https://github.com/sshpa/bayesvl>; v0.8.5; DOI:10.31219/osf.io/ya9u6.

[3] Vuong, Q. H., Ho, T. M., \& La, V. P. (2019). 'Stargazing' and p-hacking behaviours in social sciences: some insights from a developing country. European Science Editing, 45(2), 56-57.

[4] Vuong, Q. H. (2017, Dec 12). Open data, open review and open dialogue in making social sciences plausible. Nature: Scientific Data Updates. Retrieve from <http://blogs.nature.com/scientificdata/2017/12/12/authors-corner- 
open-data-open-review-and-open-dialogue-in-making-social-sciences-

plausible/> (May 30, 2019).

[5] Vuong, Q. H. (2018). The (ir)rational consideration of the cost of science in transition economies. Nature Human Behaviour, 2(1), 5, DOI: https://doi.org/10.1038/s41562-017-0281-4.

URL: https://www.nature.com/articles/s41562-017-0281-4.

[6] Ho, M.-T., La, V.-P., Nguyen, M.-H., Vuong, T.-T., Nghiem, K.-C. P., Tran, T., . . Vuong, Q.-H. (2019). Health care, medical insurance, and economic destitution: A dataset of 1,042 stories. Data, 4(2), 57; DOI: https://doi.org/10.3390/data4020057.

[7] Vuong, Q. H., La, V. P., Vuong, T. T., Nguyen, V. H., Ho, M. T., Nguyen, T. H. K., Bui, Q. K., \& Ho, M. T.. (2018). Cultural additivity: Behavioural insights from the interaction of Confucianism, Buddhism, and Taoism in folktales. Palgrave Communications, 4, 143. DOI: https://doi.org/10.1057/s41599-018-0189-2. URL: https://www.nature.com/articles/s41599-018-0189-2.

[8] Vuong, Q.-H., Bui, Q.-K., La, V.-P., Vuong, T.-T., Ho, M.-T., Nguyen, H.-K. T., . . Ho, M.-T. (2019, January 26). Cultural evolution in Vietnam's early 20th century: a Bayesian networks analysis of Franco-Chinese house designs. (Working Paper No. PKA-1901). arXiv Preprints, arXiv:1903.00817v1 [Stat.AP].

[9] Le, A.-V., Do, D.-L., Pham, D.-Q., Hoang, P.-H., Duong, T.-H., Nguyen, H.-N., ... Vuong, Q.-H. (2019). Exploration of youth's digital competencies: a dataset in the educational context of Vietnam. Data, 4(2), 69. DOI: https://doi.org/10.3390/data4020069. 